\title{
Exemples d'élimination industrielle des suspensions solides en vue du recyclage
}

\author{
J.-M. Rovel \\ Ingénieur Civil des Mines, Chef de Département \\ Société Degrémont
}

L'utilisation industrielle de l'eau n'entraîne des consommations effectives que dans quelques cas très particuliers (incorporation de l'eau aux produits finis ou plus souvent évaporation).

Dans tous les cas, l'eau ne sert que de moyen de transport, fluide de réfrigération, fluide de nettoyage... et est donc restituée au milieu naturel avec seulement une certaine dégradation de sa qualité due à l'introduction, au cours des opérations ci-dessus mentionnées, de polluants divers.

Le recyclage de l'eau est donc toujours possible, à condition soit de pouvoir, au niveau de l'utilisation, parer aux principaux inconvénients apportés par la présence de ces polluants, soit de retraiter l'effluent pour éliminer cet apport.

En général, je crois pouvoir dire que, dans la quasi totalité des cas, les techniques actuelles du traitement d'eau sont capables de faire ce retraitement et donc de pouvoir mettre en circuit fermé la plupart des usines. Toutefois, il faut reconnaitre que ceci n'est pas toujours le système le plus économique et qu'il vaut mieux généralement n'envisager que des recyclages partiels permettant d'éliminer les polluants les plus gênants, voire sélectionner les postes de travail qui peuvent demander une qualité moindre de l'eau et d'alimenter en « eau propre » les postes les plus sensibles.

Le tableau 1 essaie de schématiser les principaux inconvénients apportés par les quatre grandes catégories de pollution ci-dessus mentionnées, ainsi que les remèdes et les appareillages à mettre en ouvre pour éliminer ces polluants.

Nous avons volontairement classé ce tableau de manière à avoir, du haut vers le bas, les techniques conduisant à des coûts croissants du mètre cube d'eau recyclée.

Tableau 1

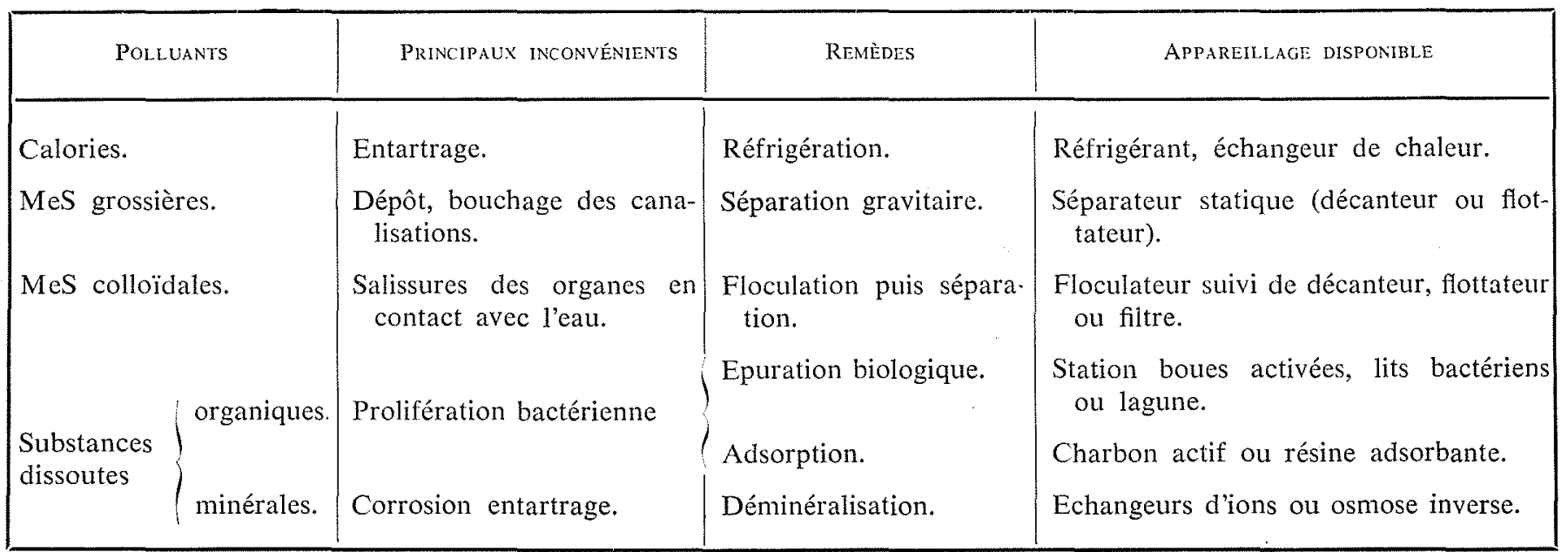


En parcourant ce tableau, on voit que si l'on voulait être exhaustif, il faudrait balayer tout l'éventail des traitements d'eau, ce qui dépasserait largement le cadre d'une telle conférence.

J'ai donc choisi de me limiter à quelques exemples tirés de l'expérience de Degrémont et qui me semblent intéressants. En effet, ils couvrent à peu près tout l'éventail die toutes les techniques utilisables. D'autre part, ils ont été introduits dans la pratique industrielle pour des raisons diverses :

\section{- Traitement des condensats des générateurs de vapeur.}

Dans ce cas, il s'agit de récupérer de très grosses quantités d'eau ultrapure, dont le traitement initial a été très coûteux. Ces traitements se sont donc très rapidement imposés, puisque le coût de «retraitement » est nettement inférieur au traitement initial (déminéralisation totale de l'eau brute).

\section{- Récupération des eaux et des fibres dans l'industrie papetière.}

Dans ce deuxième cas, il s'agit d'une industrie grosse consommatrice d'eau, mais qui, jusqu'à présent, dans les pays comme le nôtre du moins, n'avait été que médiocrement incitée à recycler l'eau puisque généralement implantée auprès de rivières assez abondantes. Par contre, la récupération concomitante des fibres permet maintenant de rentabiliser les installations de traitement, tout en allégeant le prélèvement d'eau et la pollution rejetée.

\section{- Réutilisation de l'eau après traitement biologique.}

Cette réutilisation n'est encore guère pratiquée que dans les régions où le déficit en eau est important. Toutefois, elle offre la possibilité de récupérer, derrière les traitements classiques d'eaux d'égoûts urbains, de très grandes quantités d'eau au voisinage direct de leur emploi pour l'industrie, voire même pour les usages humains.

Vous aurez, au cours de ces deux journées, d'autres exemples de recyclage, mais la liste est loin d'être exhaustive et, par exemple, notre Société a participé à la construction d'un grand nombre de stations pour des industries aussi diverses que celles des traitements de surfaces, de sucreries, de la sidérurgie, des industries chimiques ou pétrochimiques qui toutes comportent une réutilisation partielle de l'eau résiduaire après traitement.

\section{1 - Traitement des condensats des générateurs de vapeur haute pression}

Suivant le type de chaudière utilisé, les qualités et quantités d'eau mises en cuvre dans les générateurs haute pression sont très variables. Néanmoins, dans la plupart des cas, il faut maintenir, au niveau du générateur, les eaux de très haute pureté. En effet, toute introduction de matières minérales ou organiques risque de provoquer des dégâtis considérables sur les surfaces d'échange, par corrosion ou par surchauffe locale dès qu'un quelconque dépôt limite le transfert de chaleur.

La pollution apportée au cours du cycle eau-vapeur dans la chaudière et la turbine est variable suivant les matériaux utilisés, le type de chaudière et l'utilisation constante ou en régime de pointe de celle-ci. Toutefois, les apports sont toujours de deux types: a) Des produits de corrosion: oxydes métalliques, fer et cuivre en particulier, présents dans l'eau sous forme de colloïdes assez fins.

Dans ce cas, et contrairement aux règles du tableau ci-dessus et à celles que $\mathrm{M}$. Treille a pu vous donner ce matin, il n'est pas possible d'utiliser une floculation. En effet, l'on apporterait à l'eau des sels indésirables. Il faut donc utiliser un système de microfiltration permettant de retenir directement, sur des matériaux filtrants très fins, ces particules de quelques microns de diamètre. Ceci est possible en employant les filtres à précouche utilisant des précouches de cellulose ou des micro-résines inertes (polymères, granulés de 15 à 50 microns de diamètre). Cela est. par exemple, le cas de l'installation que nous avons montée pour l'Aluminium Péchiney à Gardanne où deux filtres à précouche (système Cannon, breveté Degrémont) permettent l'épuration en continu de $280 \mathrm{~m}^{3} / \mathrm{h}$ de condensats à $85^{\circ}$ (cf. fig. 1).

La durée moyenne d'une précouche est de dix jours. Son rendement de filtration est de $97 \%$, avec une teneur moyenne à l'entrée des filtres de 70 microgrammes/litre, constituée essentiellement par de l'oxyde ferrique.

Un cas plus favorable est rencontré sur certaines instaliations à haute température, où les produits de corrosion sont surtout sous forme d'oxydes magnétiques $\mathrm{Fe}_{3} \mathrm{O}_{4}$ ou plus généralement d'oxydes sous forme spinelles. Dans ce cas, l'on utilise la possibilité de rétention de ces fines particules sur des filtres magnétiques (cf. fig. 2).

L'intérêt de tels filtres réside dans leur compacité (très grande vitesse de filtration, de l'ordre de $0,3 \mathrm{~m} / \mathrm{s}$ ) et par la possibilité de traiter des condensats très chauds $\left(150^{\circ}\right.$ à $250^{\circ}$ ), ce qui permet d'améliorer d'autant le rendemont thermique du générateur de vapeur.

\section{b) Des produits minéraux dissous:}

Ceux-ci viennent surtout de fuites plus ou moins importantes au condenseur, de l'eau brute passant alors dans le circuit d'eau déminéralisée. Le problème essentiel est alors un problème de déminéralisation et, en conséquence, il faut mettre en cuvre une technique d'échange d'ions. Dans ce cas, l'on utilise des mixed-beds (lits mélangés de résines anioniques et cationiques) qui permettent donc, même à très grande vitesse, de fixer les ions introduits.

En fait, il est rare que l'on n'ait affaire qu'à l'une de ces deux pollutions. Il faut donc souvent disposer d'un moyen permettant à la fois l'élimination des produits de corrosion et des minéraux dissous. Ceci est possible, soit en combinant les deux techniques ci-dessus, ce qui est fait quand les risques de pollution des deux types sont importants, soit en utilisant des filtres à précouche garnis de microrésines actives (résines cationiques et anioniques broyées) ou en utilisant les propriétés de filtration des mixed-beds, le critère des choix entre ces techniques étant de savoir si l'on veut «privilégier» l'aspect filtration ou l'aspect déminéralisation. A titre d'exemple, le tableau 2 donne les résultats du traitement des condensats du réacteur BWR de Santa Maria de Garona où six lits mélangés, de diamètre $2400 \mathrm{~mm}$, assurent le traitement de $3300 \mathrm{~m}^{3} / \mathrm{h}$ d'eau déminéralisée en circulation dans le circuit vapeur, la pression de service étant de 21 bars, la température de $40^{\circ}$.

On constate, sur le tableau 2, que les lits mélangés permettent de maintenir une conductibilité pratiquement constante, quels que soient les apports. En outre, ils assurent une rétention importante des matières en suspension; celle-ci 


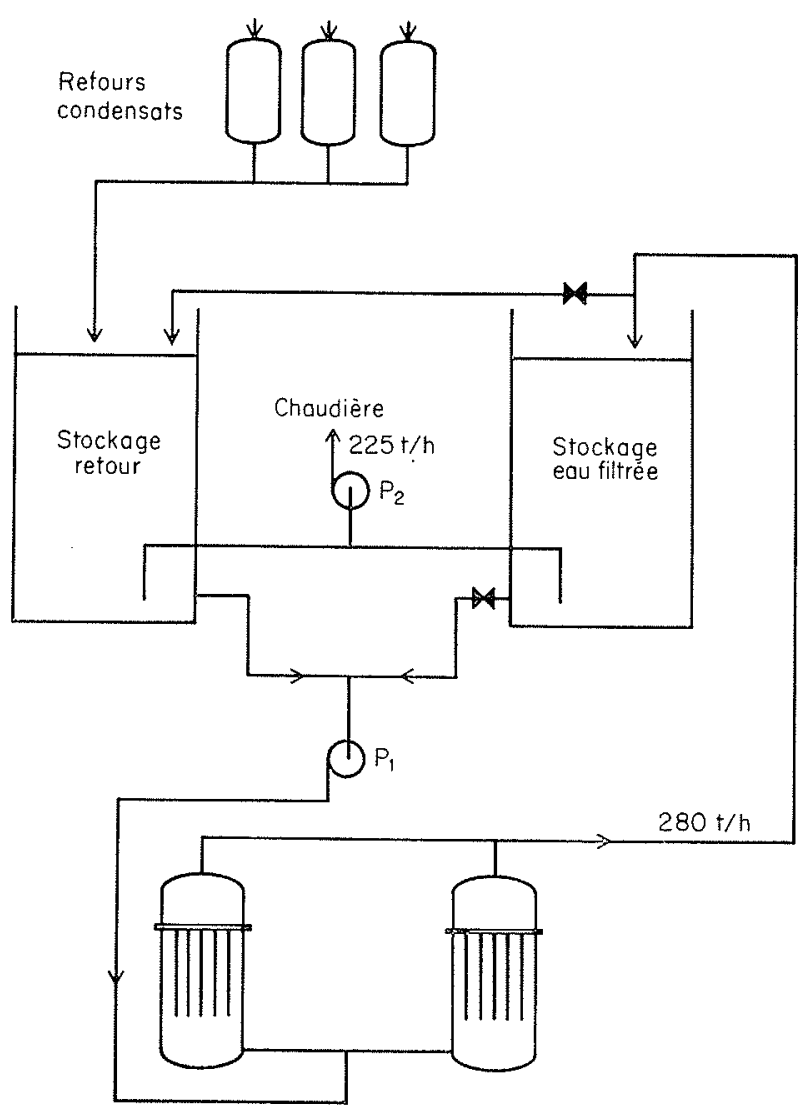

1/ Aluminium Péchiney. Gardanne. Traitement des condensats.

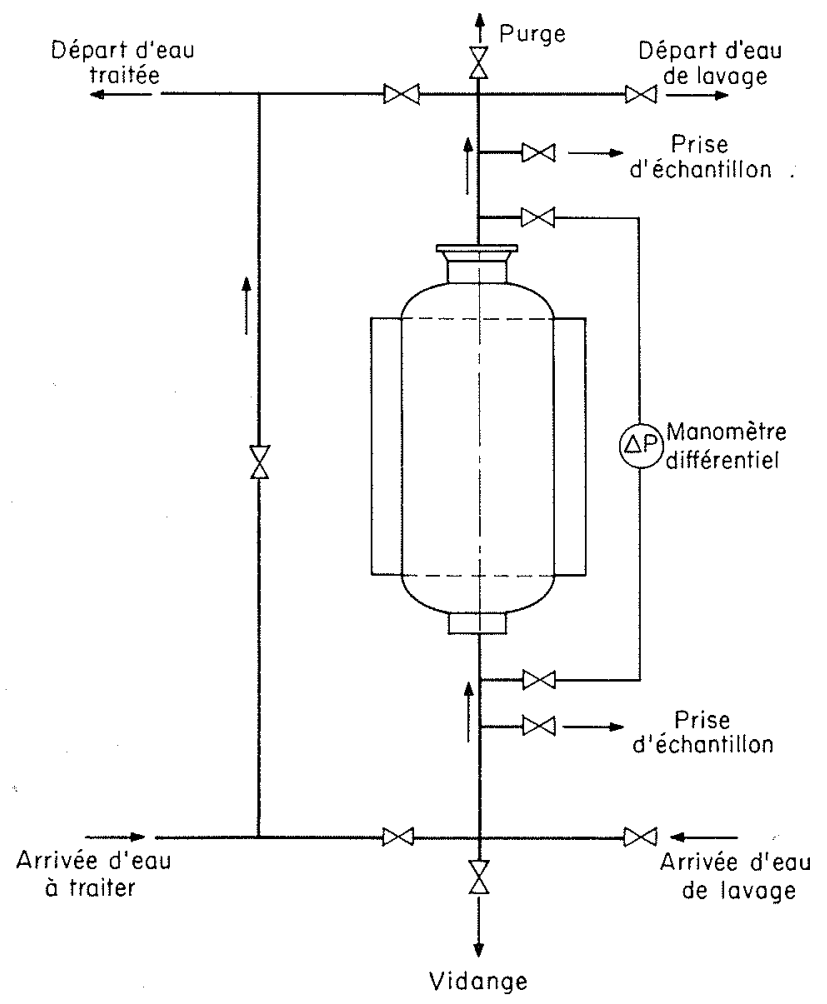

2/ Filtre électromagnétique

\section{Tableau 2}

\section{RÉSULTATS}

Traitement des condensats réacteurs BWR à Santa Maria de Garona (Espagne).

6 lits mélangés:

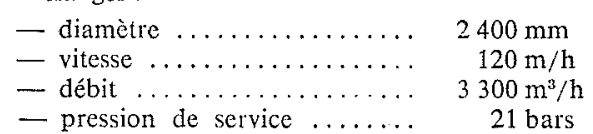

\begin{tabular}{|l|l|r|r|}
\hline \multicolumn{2}{|c|}{$\begin{array}{c}\text { Conductibilité } 2{ }^{\circ} \mathrm{C} \\
(\mu \mathrm{S} / \mathrm{cm})\end{array}$} & \multicolumn{2}{|c|}{$\begin{array}{c}\text { Matières EN SUSpension } \\
(\mathrm{ppb} \text { *) }\end{array}$} \\
\hline Entrée & Sortic & Entrée & Sortie \\
\hline 0,1 & 0,08 & 17 & 11 \\
0,1 & 0,08 & 15 & 8 \\
0,2 & 0,08 & 14 & 12 \\
0,22 & 0,085 & 186 & 16 \\
0,09 & 0,08 & 18 & 8 \\
0,15 & 0,08 & 465 & 224 \\
0,25 & 0,085 & 284 & 150 \\
0,45 & 0,12 & 240 & 21 \\
0,15 & 0,10 & 1933 & 720 \\
\hline$* 1 . \mathrm{ppb}=1 \mathrm{mg} / \mathrm{m} *$ & & \\
\hline
\end{tabular}

ne peut toutefois pas se comparer à celle obtenue sur les filtres à précouche qui, dans de tels cas, pourraient garantir moins de $10 \mathrm{ppb}$ de matières en suspension résiduelles.

\section{2 - La récupération des eaux et des fibres dans l'industrie papetière}

Les unités de production du papier sont très grosses utilisatrices d'eau : $50 \mathrm{~m}^{3} /$ tonne de papier journal ou kraft, 150 à $200 \mathrm{~m}^{3} /$ tonne de carton, papier magazine ou papier fin, sont des normes courantes. En effet, l'eau sert de véhicule aux éléments constitutifs du papier: fibres, charges et adjuvant.

Au cours du procédé, l'eau de process se charge essentiellement :

- de fibres de tailles variées, allant de l'état quasi colloïdal à des tailles suffisantes pour décanter très rapidement;

- de «charges », en général très fines: carbonate de calcium, kaolin, etc., toujours à l'état colloïdal;

- de minéraux dissous, par exemple: les ions sulfatés apportés par le sulfate d'aluminium souvent utilisé en encollage sur machine; 


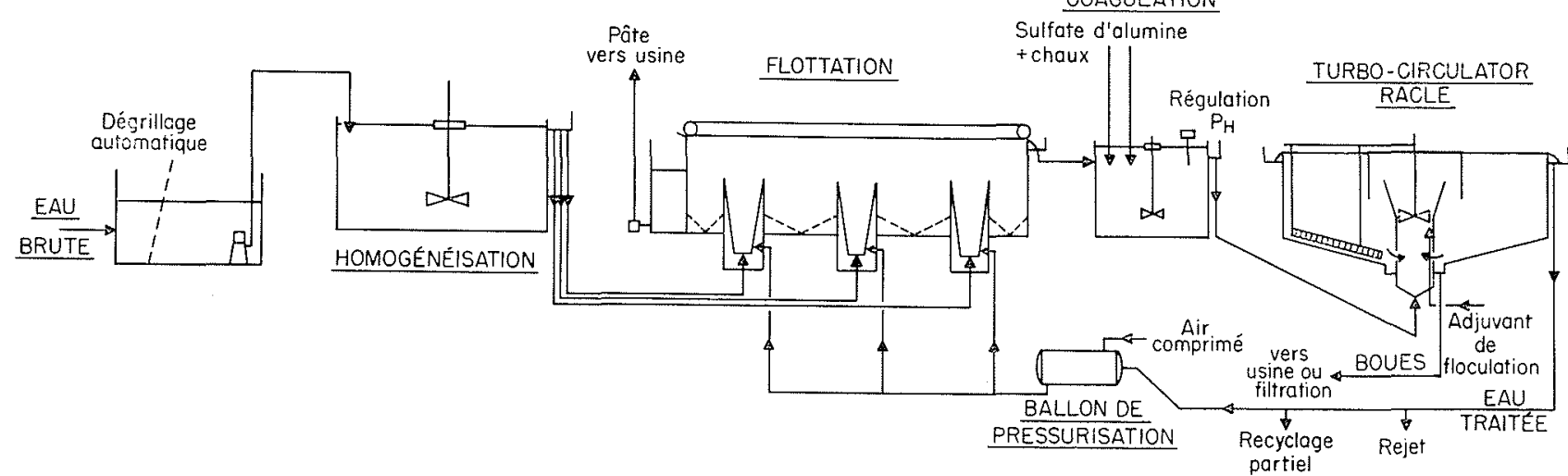

STATION A

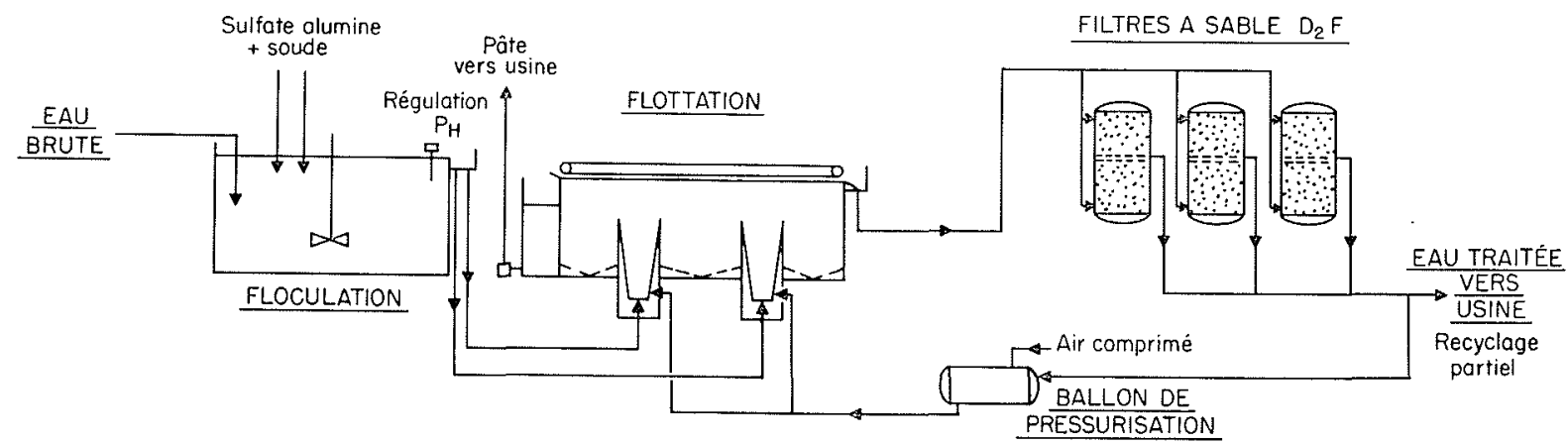

3/ Exemple de chaine de traitement en papeterie,

permettant un recyclage partiel des matières et de l'eau

Tableav 3

\begin{tabular}{|c|c|c|c|c|c|c|c|c|c|c|c|}
\hline \multicolumn{2}{|c|}{$\begin{array}{l}\text { - Débit } 300 \mathrm{~m}^{3} / \\
\text { - Floculation }\end{array}$} & \multicolumn{4}{|c|}{$\begin{array}{l}\text { - Flottateur } 2 \text { têtes } \\
-3 \text { filtres D2F }\end{array}$} & \multicolumn{6}{|c|}{$\begin{array}{c}\text { Flottateur }+ \text { Turbocirculator } \\
\text { (avec floculation } 350 \mathrm{ppm} \text { S.A.; chaux } 1 \text { à } 2 \mathrm{ppm} \text { polyélectrolyte) }\end{array}$} \\
\hline & $\begin{array}{c}\text { ENTRÉE } \\
\text { StATION } \\
\text { Circuit } \\
\text { ouvert }\end{array}$ & $\begin{array}{c}\text { Sortie } \\
\text { StATION } \\
\text { Circuit } \\
\text { ouvert }\end{array}$ & $\begin{array}{c}\text { ENTRÉE } \\
\text { STATYON } \\
\text { Recyclage } \\
80 \%\end{array}$ & $\begin{array}{c}\text { SORTIE } \\
\text { FLOTTATEUR }\end{array}$ & Filtres & & $\begin{array}{c}\text { ENTRÉE } \\
\text { Station } \\
\text { Circuit } \\
\text { ouvert }\end{array}$ & $\begin{array}{c}\text { Sortie } \\
\text { Station } \\
\text { Circuit } \\
\text { ouvert }\end{array}$ & $\begin{array}{c}\text { ENTRÉE } \\
\text { Station } \\
\text { Recyclage } \\
65 \%\end{array}$ & $\begin{array}{c}\text { SORTIE } \\
\text { FLOTTATEUR }\end{array}$ & $\begin{array}{c}\text { SORTIE } \\
\text { DÉCANTEUR }\end{array}$ \\
\hline $\mathrm{pH}$ & 7 & 7 & 6 & 7 & 7 & $\mathrm{pH}$ & 7 & 7 & 6,5 & 6,5 & 7 \\
\hline $\mathrm{DCO}_{\mathrm{at} 2}$ & 350 & 300 & 1600 & 1500 & - & $\mathrm{DCO}_{\mathrm{at2}}$ & 900 & 380 & 1570 & 1500 & 950 \\
\hline $\mathrm{DBO}_{\mathrm{ad} 2}$ & 140 & 120 & 600 & 560 & 一 & DBO & 380 & 160 & 660 & 640 & 400 \\
\hline $\mathrm{SO}_{4}^{--}$ & 560 & 580 & 2500 & 2500 & - & $\mathrm{SO}_{4}$ & 20 & 220 & 385 & 385 & 590 \\
\hline $\mathrm{Cl}^{--}$ & 65 & 65 & 310 & 310 & - & $\mathrm{Cl}^{--}$ & 71 & 71 & 180 & 180 & 180 \\
\hline $\mathrm{MeS}$ & 300 & $10-15$ & 300 & $40-60$ & 15 & $\mathrm{MeS}$ & 1300 & 25 & 1500 & 500 & 25 \\
\hline
\end{tabular}


- de substances organiques colloïdales, exemple amidons, ou dissoutes, exemple colles ou dérivés solubles de la lignine amenés par la pâte, en particulier quand celle-ci n’a pas été blanchie.

Comme indiqué ci-dessus, le problème du recyclage en papeterie se pose très différemment suivant que l'on veut ou que l'on peut envisager de recycler l'eau seulement ou l'eau et les fibres, voire l'eau et l'ensemble fibres plus charges. En effet, l'on peut, selon le cas, songer aux schémas suivants :

\section{Récupération des fibres seules}

Utilisation de filtres (en général, filtres à tambour rotatii ou à disque) ou de flottateurs sans emploi de floculant.

Dans les deux cas, ces techniques permettent de récupérer entre 60 et $85 \%$ des matières en suspension. Elles ont toutes deux l'avantage de recirculer très rapidement les fibres en fabrication et donc de ne poser aucun problème de qualité en cas de fabrications variées sur la machine.

\section{Récupération fibres plus charges}

Dans ce cas, il faut floculer l'eau afin de récupérer les fibres les plus fines, mais également les charges organiques ou minérales à l'état colloïdal. La séparation sera faite ensuite suivant le rapport fibres/charges dans des flottateurs ou des décanteurs en général à circulation de boues.

La figure 3 montre deux types de chaînes utilisés dans différentes papeteries en France.

Le tableau 3 regroupe les résultats obtenus sur les deux chaînes de la figure 3 et montre l'efficacité de l'épuration acquise sur les installations de:

- Station A: papeterie utilisant des vieux papiers, d'un débit de $300 \mathrm{~m}^{3} / \mathrm{h}$ et équipée d'une station comportant une floculation avec 30 à $60 \mathrm{ppm}$ de sulfate d'alumine, $1 \mathrm{ppm}$ de polyélectrolyte (quantité nettemest insuffisante pour floculer l'ensemble des colloïdes présents), un flottateur de $60 \mathrm{~m}^{2}$ et une batterie de filtres D2F.

- Station B : également une papeterie utilisant des vieux papiers avec une récupération de fibres sur un flottateur de $250 \mathrm{~m}^{3} / \mathrm{h}$ suivi d'une floculation et d'une décantation dans un décanteur à recirculation de boues (Turbocirculator) employant $350 \mathrm{ppm}$ de sulfate d'alumine et 1 à 2 ppm de polyélectrolyte.

Dans les deux cas, les deux premières colonnes donnent les résultats obtenus en circuit ouvert, les troisième, quatrième et cinquième ceux obtenus après un recyclage en fabrication, respectivement de 80 et $65 \%$ de l'eau.

\section{Récupération de l'eau}

Dans tous les cas, la récupération de l'eau est possible, mais suivant que l'on a utilisé une floculation plus ou moins poussée, cette eau pourra être recyclée directement après le séparateur ou nécessitera une filtration préalable, afin de ne pas risquer des bouchages des organes les plus sensibles tels que les rampes assurant le nettoyage des cylindres presseurs.

En fait, si cette recirculation d'eau est toujours possible, il est évident que les moyens cités ci-dessus ne permettent

\section{Tableau 4}

\section{EXEMPLE STATION B}

\begin{tabular}{|l|c|c|c|}
\hline & DÉBIT & SO $_{4}$ & DCO \\
\cline { 2 - 4 } Circuit ouvert & $50 \mathrm{~m}^{3} / \mathrm{t} \mathrm{ppi}$ & 190 & 380 \\
\hline $50 \%$ recyclage & $25 \mathrm{~m}^{3} / \mathrm{t}$ & 320 & 750 \\
\hline $80 \%$ recyclage & $10 \mathrm{~m}^{3} / \mathrm{t}$ & 750 & 1500 \\
\hline $100 \%$ recyclage & $1,3 \mathrm{~m}^{3} / \mathrm{t}$ & $4000 *$ & 9500 \\
\hline Précipitation $1100 \mathrm{ppm}$. & & \\
\hline
\end{tabular}

d'éliminer qu'une partie de la pollution introduite par la fabrication, à savoir les matières en suspension ou colloïdales, mais quills laissent la quasi totalité des matières organiques dissoutes. Aussi, celles-ci auront tendance à se concentrer progressivement comme le montre le tableau 4 représentant le cas de l'unité $B$.

En fait, seuls les trois premiers points ont été expérimentalement vérifiés, la dernière ligne représentant un calcul de l'état auquel on arriverait si l'on utilisait un recyclage de $100 \%$. On constate que ceci imposerait de prévoir des mesures très sévères pour contrôler la corrosion (en pratique, circuit tout en inox ou plastique) et l'entartrage étant donné l'accroissement des concentrations et de la température (précipitation de carbonate et sulfate de calcium).

Dans la plupart des cas, il sera donc plus prudent de se limiter à une recirculation allant de 60 à $85 \%$ qui, pratiquement, n'a que des avantages, sauf à la rigueur les problèmes de développement bactérien favorisé par l'augmentation de la pollution organique dissoute (cf. tableau 3) ct une légère augmentation de la corrosivité.

\section{3 - Réutilisation de l'eau après traitement biologique}

Cette réutilisation n'existe encore que très peu en France, si ce n'est clandestinement par l'intermédiaire de toutes nos rivières, mais elle est déjà pratiquée à une grande échelle dans des pays plus arides que le nôtre, tels que les U.S.A., le Japon, Israël ou même la Grande-Bretagne.

A l'aval d'une station conventionnelle de traitement biologique d'eaux urbaines, il reste dans l'eau quelques éléments ayant échappé au traitement biologique ou à la clarification subséquente, des éléments dissous non biodégradables, formant une DCO résiduelle d'environ $100 \mathrm{mg} / \mathrm{l}$, ainsi que différents sels dont les plus gênants, pour les emplois industriels, sont souvent les composés du phosphore et de l'azote qui conduisent fréquemment à des développements très rapides d'algues ou de bactéries dans les circuits.

Le tableau 5 donne une analyse typique d'une telle eau. 
Tableau 5

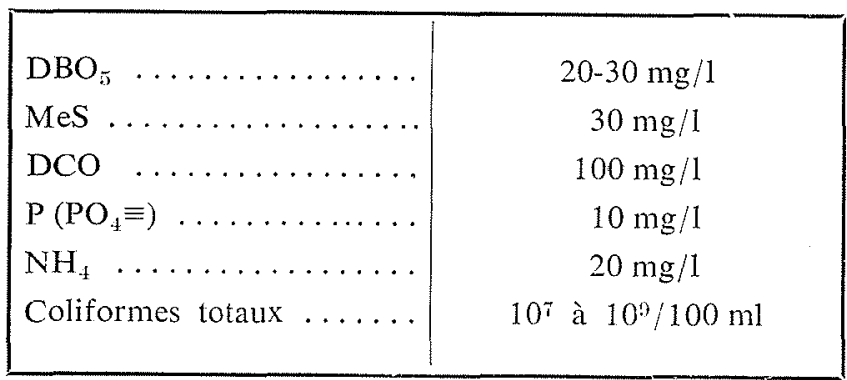

Aussi, suivant les applications, deux grands types de «traitement tertiaire » sont employés :

a) Les traitements permettant d'éliminer les matières en suspension et les risques de prolifération bactérienne. A ce titre, on utilise surtout des procédés de filtration :

- soit sur sables avec des filtres à haute couche $(1$ à $2 \mathrm{~m})$ et à grande vitesse $(10$ à $30 \mathrm{~m} / \mathrm{h}$ ) qui éliminent entre 50 et $80 \%$ des matières en suspension ainsi que 30 à $50 \%$ de la DBO résiduelle;

La figure 4 précise l'influence de la hauteur de couche, de la granulométrie du sable et de la vitesse de filtration sur les rendements d'élimination des matières en suspension.

- soit sur un matérial poreux qui permettra une colonisation de ses pores par une culture bactérienne et qui adjoindra, à l'effet de filtration, une dégradation biologique plus poussée de l'effluent. Par exemple, en utilisant un filtre chargé de biolite (chamotte spécialement développée par Degrémont), pour une hauteur de couche de $1,50 \mathrm{~m}$ et une vitesse de $10 \mathrm{~m} / \mathrm{h}$, on a obtenu une réduction des matières en suspension de $90 \%$ et de $60 \%$ de la DBO.

Après de telles filtrations, il faut en général stériliser l'eau par adjonction de chlore, afin d'éviter les risques de contamination des circuits aval. Une telle eau est parfaitement

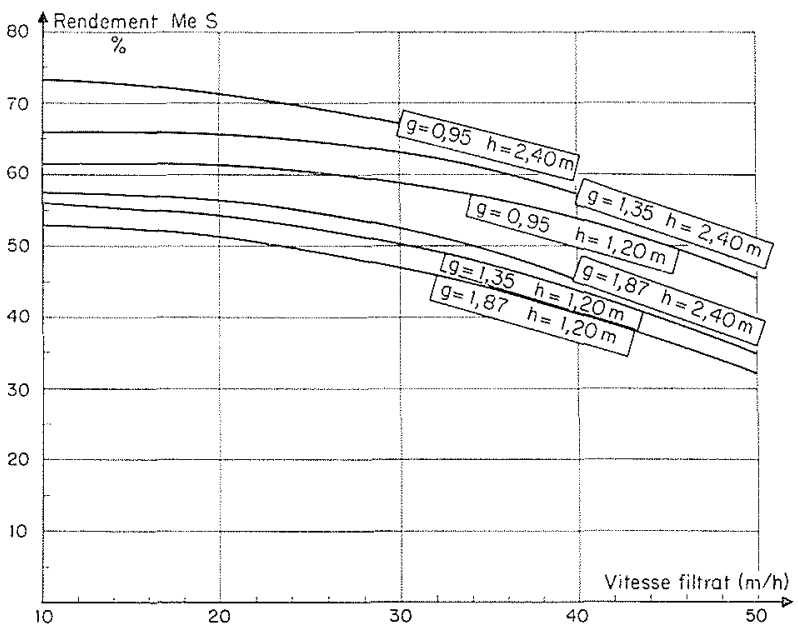

4/ Filtration sur sable d'effluent secondaire de station d'eaux résiduaires urbaines.

apte à de nombreux usages industriels (lavages, refroidissements...)

b) Dans le cas où l'on veut obtenir une épuration beaucoup plus poussée pour des usages plus nobles, on peut envisager de refaire une eau de qualité pratiquement potable, ce qui est fait, par exemple, dans l'installation américaine du lac Tahoé où l'eau est traitée successivement :

- par de la chaux à pH élevé, ce qui assure une floculation et la précipitation des phosphates:

- par un stripping à l'air qui élimine l'essentiel de l'ammoniaque;

- par filtration sur sable, puis sur filtre à charbon actif, qui adsorbe les matériaux organiques dissous qui se sont révélés être réfractaires au traitement biologique;

- enfin, l'effuent est chloré.

Le tableau 6 donne les résultats obtenus sur cette station. On peut y noter que la qualité finale est supérieure à celle de bien des eaux de surfaces de nos rivières françaises.

\section{Tableau 6}

LaC TAHó́ : Evolution de L'EAU SUIVANT LE TRAITEMENT

\begin{tabular}{|c|c|c|c|c|c|}
\hline & EAU brute & $\begin{array}{l}\text { APRÈS ÉPURATION } \\
\text { BIOLOGIQUE }\end{array}$ & $\begin{array}{l}\text { APrès } \\
\text { DÉSAMMONISA TION }\end{array}$ & APrÈs FILTRATION & $\begin{array}{l}\text { ApRÈs } \\
\text { CHARBON ACTIF } \\
\text { ET CHLORATION }\end{array}$ \\
\hline DCO $\ldots \ldots \ldots \ldots \ldots(\mathrm{mg} / 1)$ & $200-500$ & $60-125$ & 25 & 20 & 10 \\
\hline $\mathrm{DBO}_{\overline{5}} \ldots \ldots \ldots \ldots$ & $200-400$ & $20-50$ & $5-10$ & 4 & 1 \\
\hline $\operatorname{MeS} \ldots \ldots \ldots \ldots \ldots(\gg)$ & 225 & $10-30$ & $5-10$ & 0,9 & 0,9 \\
\hline$\ldots \ldots \ldots \ldots \ldots \cdots(»)$ & $10-15$ & & 5,7 & 0,8 & 0,8 \\
\hline $\mathrm{N}$ ammoniacal ........ $(»)$ & $20-30$ & $20-30$ & 5- 9 & $5-9$ & Chloramines \\
\hline $\begin{array}{c}\text { Bactéries coliformes } \mathrm{MP} \\
\text { totaux } \ldots \ldots \ldots(\mathrm{N} / 100 \mathrm{ml})\end{array}$ & $\sim 10^{\mathrm{s}}$ & & & & $<2$ \\
\hline
\end{tabular}




\section{Conclusion}

Je pense, par les quelques exemples ci-dessus mentionnés, avoir montré comment, à partir de la connaissance des éléments polluants apportés au cours de l'utilisation de l'eau, on peut adapter un traitement qui permet l'élimination de ces différents polluants.
Des considérations d'ordre économique conduisent, en général, à limiter le recyclage afin d'éviter d'avoir à éliminer les matières organiques et surtout minérales dissoutes. Néanmoins, notre expérience nous a montré que, pour presque tous les utilisateurs industriels d'eau, des recirculations d'au minimum $50 \%$ de leur débit étaient envisageables et que, dans de nombreux cas, l'on pouvait même aller jusqu'à 85 , voire $90 \%$.

\section{Discussion}

Présidcht: M. P. BERGERON

M. le Président remercie M. Rover pour son exposé qui présente une bonne synthèce des procédés utilisés dans ce domaine et ouvre la discussion.

M. J. THÉVENIN (Agence Financière de Bassin Seine-Nomandie) intervient en ces termes:

M. Rover a cité un certain nombre de cas, choisis hors de France, où les eaux usées régénérées sont réutilisées pour les besoins de lindustrie el même pour lalimentation en eau potable. Je donnera au moins un exemple en France: celui de la ville du Havre, qui vend aux industries du secteur portuaire tout ou partie des effucnts sortant de la station d'épiration.

En effet, répond M. Rovel, ces eaux sont distribuées par un "réseau séparé » et utilisées par les industriels pour la réfrigération et le lavage.

Il y a aussí en cours d'étude - même en France - des projets très limités de réinjection de telles eaux dans des nappes en vue de leur réutilisation ultérieure dans des distributions d'eau potable.

Ces caux subissent-elles un traitement suffisant - avant d'être envoyées dans les réseaux de distribution d'eau potable - pour que tont danger d'origine microbienne soit écarté ? demande M. Misson.

Si la «stérilisation» est bien faite et parfaitement contrôlée, répond M. Rovel, il n'y a guère de danger sur le plan microbien car les traitements «microbicides» et «virocides» sont bien connus. Pour lui, le danger le plus grave des eaux ainsi recyclées réside dans certaines substances minérales et surtout organiques existant à l'étai de traces - très difficiles à détecter - et dont on peut toujours soupçonner qu'elles pourraient être néfastes pour la santé publique.

On est sûr de pouvoir contrôler l'importance de ces traces ai niveau du p.p.m. (partie par million) mais pour certains produits de synthèse, hydrocarbones pesticides ou herbicides, ne conviendraitil pas d'aller plus loin?

Un auditeur estime plus satisfaisant de réinjecter dans la nappe des eaux d'égout convenablement traitées que de les restituer directement à la rivière. On peut penser, qu'au cours de leur long parcours souterrain, une épuration naturelle améliosera la qualité de ces ealux.

Ces mécanismes existent en effet, mais il niest pas facile de contrôler leur efficacité et leur pérennité (risque de saturation...) a!ıssi, si la nappe intéressée est vraiment très propre, répond M. Rovel, cette réinjection n'est peut-être pas souhaitable; ne vaut-il pas mieux rester maître du processus d'épuration?

Avez-vous, demande M. Chevalier, une expérience des traitemenls électriques tels que l'électro-flottation ou l'électro-floculation?

Oui, répond $\mathrm{M}$. Rover, l'électro-flottation est un système de fottation parni dautres, intéressant surtout dans le cas de très petits débits. Il consiste à engendrer, par électrolyse de l'eau, des bulles d'oxygène et d'hydrogène qui s'accrochent au «floc» et le font monter à la surface.

Par contre, je vous ai montré quelques photos de flottateur à air dissous de grandes tailles; dans ces appareils, on engendre les bulles par le processus suivant: on sature d'abord l'eau sous une pression d'air de 5 on 6 bars; ainsi, l'eau contient 5 à 6 fois plus d'air dissous qu’à la pression atmosphérique; on produit la désorption de cet air en ramenant l'eau à cette dernière pression; on obtient ainsi, au sein du liquide, des bulles qui ont à peu près le même diamètre que celles réalisées dans les procédés d'électro-flottation et donc le même effet de séparation des flocs.

L'électro-floculation est basée sur un principe tout différen...

M. Trenlle vous a parlé, ce matin, des problèmes de stabilité des collö̈des liés aux charges électriques qui entourent ces colloïdes ct qui les empechent de floculer.

Le procédé d'électro-floculation consiste en une électrolyse de leau résiduaire qui permet la déstabilisation de ces colloüdes et leur rassemblement en combinant des effets purement électriques et d'autres chimiques (introduction d'ions $\mathrm{Al}$ ou $\mathrm{Fe}^{++}$et $\mathrm{Fe}^{++4+}$ par dissolution d'anodes en $\mathrm{Al}$ ou $\mathrm{Fe}$ ).

C'est donc d'abord un procédé de coagulation-floculation qui a des avantages annexes tels la décoloration... dus à l'oxydation anodique de certains composants.

11 est évident que ces deux procédés: flottation, électro-coagulation, ont leur place dans les systèmes décrits ci-dessus.

M. le Président clôt la discussion en remerciant $M$. Rovel et les personnes aui ont animé le débat. Il donne ensuite la parole à M. Serpaud pour lexposé de la communication qu'il a établie en collaboration avec M. LEJEUNE. 


\section{Abstract \\ Examples of industrial separation of suspended solids for recycling}

Once he is familiar with the polluants introduced into the water by the process the water treatment expert is able to eliminate them and return water of good quality to the process.

Pollution is subdivided into four major categories (Table 1) in an attempt to schematize the principal methods and instruments required to eliminate pollutant.

As a general rule, recycling is limited by economical tather than technical factors. This is illustrated by the three following examples:-

\section{TREATMENT OF STEAM GENERATOR CONDENSATE}

For this application, recirculation of very pure boiler circuit (turbine) water costs much less than surface water treatment.

The most suitable methods for various input products corrosion products and/or dissolved salts) are discussed, e.g. pre-coat filters, magnetic filters, mixed beds.

\section{FIBRE AND WATER RECOVERY IN THE PAPERMAKING} INDUSTRY

The amount of water the paper mills draw from the natural medium has to be limited and the maximum possible quantity of valuable effluent constituents recycled (fibres alone or with fillers depending on manufactured paper properties).

Two specific experiments show how "purification circuits" can be adapted to the problem.
Diagram 3 shows the two processing stations used, both of which allowed recovery of the fibre and all or part of the fillers with approximately 75 per cent of the water. Methods employed were flocculation followed by sedimentation, flotation or filtration according to the products to be eliminated, with special consideration of the fibre/fillers ratio.

Table 4 shows how concentration in the circuit varies with gradual closure of the latter. An increase in the concentration of dissolved organic and mineral matter is seen to limit possibie circuit closure.

\section{RE-USE OF WATER AFTER BIOLOGICAL TREATMENT}

Re-use of water can be a particularly attractive proposition for such "routine" purposes as cooling and soil washing. This provides users with substantial local water supplies at low cost after conventional domestic sewage treatment, adequate filtration and sterilization.

More "noble" recycling applications on the other hand require sophisticated equipment for such chemical and physical processes as phosphate precipitation, elimination of ammonia, efficient, thorough filtration, also adsorption on activated carbon followed by sterilization.

The water thus obtained has similar properties to those of drinking water and is undoubtedly better than most of the surface water available in industrialized countries. 